\title{
Simulation of injury potential compensation by direct current stimulation in rat spinal cord
}

\author{
Aihua Wang, Guanghao Zhang, Cheng Zhang, Changzhe Wu, Tao Song and Xiaolin Huo* \\ Beijing Key Laboratory of Bioelectromagnetism, Institute of Electrical Engineering, Chinese Academy \\ of Sciences, No. 6 Beiertiao, Zhongguancun, Haidian district, Beijing 100190, China
}

\begin{abstract}
Injury potential, a significant index of spinal cord injury (SCI), is generated by the movement of extracellular ions. It can be compensated through applied direct current (DC) stimulation, which prevents the influx of the free calcium, and eventually reduces the development of secondary injury. Therefore, the compensation of injury potential is beneficial to the repairing of the function of spinal cord. The compensation effect can be evaluated by whether the magnitudes of longitudinal electric fields (EFs) are compensated to zero. However, there have been no established criteria to determine the distribution and shape of stimulating electrodes. In this study, in order to optimize the stimulating electrodes, a finite element model (FEM) of rat spinal cord was developed, and the EFs changes induced by electrodes of different sizes, shapes and locations after SCI were calculated. All the designed configurations of electrodes were able to compensate injury potential, but the resultant compensation effects vary. Pin and disc electrodes produced uneven EFs, while ring electrodes produced uniformly distributed EFs. Moreover, large ring electrodes can compensate the longitudinal EFs almost to zero with relatively low current density $\left(0.55 \mu \mathrm{A} / \mathrm{mm}^{2}\right)$ applied. These results provide a basis for the determination of electrical stimulation parameters in the compensation of injury potential.
\end{abstract}

Keywords: Direct current stimulation, finite element model, spinal cord injury, electric fields

\section{Introduction}

Injury potential, direct current (DC) potential gradient between intact section and injured site, is an important indicator of microenvironment changes of damaged spinal cord. Its importance was not recognized until Borgens et al. studied the injury currents after spinal cord injury (SCI) [1]. Since then, injury potentials in rat, cat and guinea pig have been investigated [2-5]. All these researches indicated that injury potential is formed immediately after SCI. Further study showed that there is a positive relationship between the initial amplitude of injury potential and the grade of injury [6].

Injury potential is generated by the movement of extracellular ions, which are mainly $\mathrm{Na}^{+}$and $\mathrm{Ca}^{2+}$. $\mathrm{Ca}^{2+}$ is the major initiator of secondary injury process. Therefore, an effective method for SCI repairing is to immediately prevent the influx of the free calcium after injury. Our research team put forward an

\footnotetext{
*Corresponding author: Xiaolin Huo, Beijing Key Laboratory of Bioelectromagnetism, Institute of Electrical Engineering, Chinese Academy of Sciences, No. 6 Beiertiao, Zhongguancun, Haidian district, Beijing 100190, China. Tel.: 86-10-82547242; Fax: 86-10- 82547242; E-mail: huoxl@ mail.iee.ac.cn.
} 
approach to compensate injury potential through applied direct current electrical stimulation. Complete compensation can reduce the injury potential to 0 . The effect of this approach had been verified by in vivo experiment in rat [7]. Results showed that through stimulation, the initial amplitude of injury potential is significantly reduced. This can be attributed possibly to the prevention of $\mathrm{Ca}^{2+}$ influx and a certain self-repair of the injured axonal membrane. In order to verify the concentration variance of extracellular and intracellular ions, it is necessary to study the electric field distributions before and after the compensation of injury potential. This can be done using the double sucrose gap technique, which provides a simplified controllable in vitro environment [8].

Double sucrose gap recording chamber consists of three large compartments and two small gaps. The central large compartment contains Krebs solution, the function of which is similar to that of cerebrospinal fluid (SCF). While the two side large compartments contain $120 \mathrm{mM} \mathrm{KCl}$. The two small gaps, located on the two sides of the central compartment, contain $230 \mathrm{mM}$ sucrose. Using this chamber, the compound action potential, an index of the integrity of the cell membrane, can be measured by the extracellular microelectrodes. Existing studies that use this double sucrose gap chamber mainly focus on the effectiveness of repairing SCI by different membrane repair materials [8-10]. There is no established result showing interest in changing the direction of ion flow by electric stimulation.

In the present study, the double sucrose gap recording chamber was used to measure and compensate injury potential. In order to compensate injury potential, the compensation parameters should be determined. A three-dimensional (3D) finite element model (EFM) of rat spinal cord, comprising the C3 to L1 segments, was established to investigate the magnitude and distribution of longitudinal electric fields (EFs), the main factors of EFs that influence the movement of $\mathrm{Ca}^{2+}$. Different sizes, geometries and locations of electrodes were tested until the prospective compensation effect was achieved.

\section{Materials and methods}

\subsection{Construction of model}

According to the anatomy, the spinal cord comprises dura matter, SCF, white matter and grey matter. Since the dura was removed before the spinal cord was fixed in the recording chamber (Figure 1(b)), the model only consists of white matter, grey matter and SCF, and SCF was substituted by Krebs solution. The bottom surface of the double sucrose gap recording chamber and the mid-sagittal spinal cord plane were set as XOY plane and XOZ plane, respectively. The origin of the coordinate is the center of the bottom surface of the recording chamber. Therefore, the model is symmetric about the $\mathrm{XOZ}$ and $\mathrm{YOZ}$ planes, thus only a quarter of the model was used for simulation.

The boundary of the model was confirmed by the shape of the recording chamber. The transverse plane of the spinal cord was as shown in Figure 1(a), the outlines of white matter and grey matter were obtained from the MRI image of a 77 days old female rat [11]. Considering the size of the recording chamber, the spinal cord used in the experiment was the $\mathrm{C} 3$ to L1 segment. SCI occurred at the midpoint of the spinal cord within the central compartment (i.e. T3 segment). As the width of the T3 segment is 3 $\mathrm{mm}$, the width of the transverse plane should be proportionally increased to $1.5 \mathrm{~mm}$. Finally, the shape was stretched $16.5 \mathrm{~mm}$ to produce the 3D simulation structure (Figure 1(c)-1(e)).

The SCI was produced by a standardized crush to the isolated spinal cord with a Plexiglas rod. The end of the rod supplied a compression surface of $2.5 \mathrm{~mm}$ along the length of the tissue, and a transverse width of $12 \mathrm{~mm}$. During the experiment, the morphology of the spinal cord at the injury site changed. A 

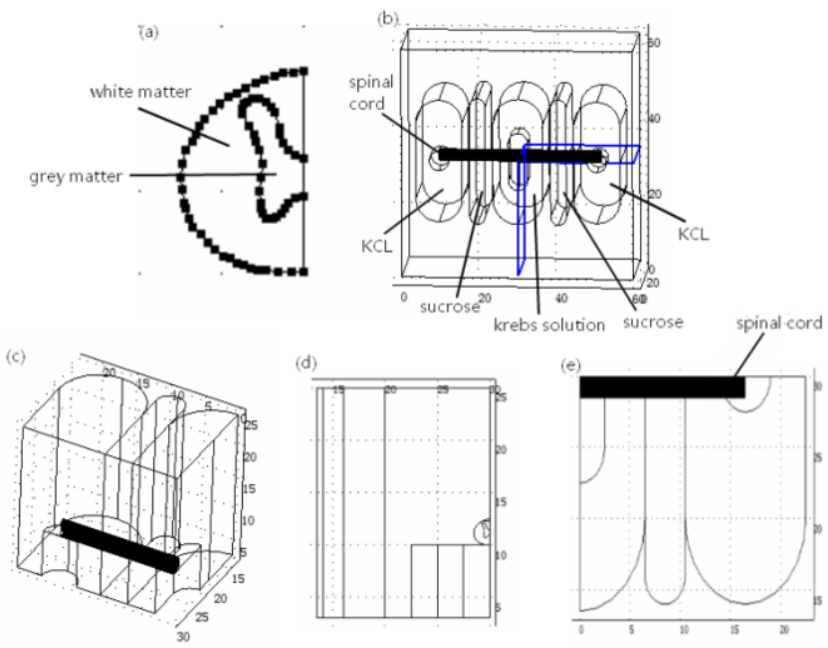

Fig. 1. Double sucrose gap recording chamber and finite element model. (a) Drawing of the transverse plane of rat spinal cord. (b) Illustrations of the double sucrose gap recording chamber, the quarter part of the chamber that was divided by the blue plane was used for the simulation. (c)-(e) Illustrations of the simulation model's shape and the tissues in oblique, transverse-sectional and top views, respectively. The dimension of the chamber is marked in the picture, in the unit of $\mathrm{mm}$.

hollow about $0.2 \mathrm{~mm}$ in depth appeared at the injury site of the spinal cord. In order to simplify the model, the distortion of the spinal cord was neglected and the conductivity of the spinal cord tissue at the injury site was simply replaced with the SCF.

\subsection{Electrical conductivities for stimulation}

The electrical conductivities of the tissues in the simulation model are shown in Table $1[12,13]$.

\subsection{Electrodes and current densities for stimulation}

Metals are commonly used as electrodes for neurostimulation. The conductivity of the electrodes was considered as $10^{7} \mathrm{~S} / \mathrm{m}$. Firstly, the compensation of injury potential was studied with a pin electrode, which had an area of $0.1256 \mathrm{~mm}^{2}$ and a length of $1 \mathrm{~mm}$. The anode was located above the injury site and the cathode set above the spinal cord in the central compartment near the sucrose gap. This electrode configuration will be referred to hereinafter as the pin configuration. Then, the compensation of injury potential by disc electrodes was studied. Four different configurations were considered for disc electrodes. In disc1 and disc 2 configurations, the position of electrodes was the same as that in the pin configuration. In disc3 configuration, the electrode was located perpendicular to that in disc1 configuration. Disc4 configuration combined the disc1 and disc3 configurations. The electrodes in disc1, disc 3 and disc 4 configurations were of the same type, which had a circular surface with a diameter of 1 $\mathrm{mm}$, and the electrode in disc 2 configuration had a diameter of $2 \mathrm{~mm}$. All electrodes in the disc configurations were $0.2 \mathrm{~mm}$ in thickness. The final experiment of injury potential compensation was performed using ring electrodes, which encircle the spinal cord. The ring electrodes were rolled with metal sheet $(0.1 \mathrm{~mm}$ thick) based on the diameter of the spinal cord. Two different types of ring electrodes were made, one with a width of $0.5 \mathrm{~mm}$, while the other with a width of $1 \mathrm{~mm}$, forming ring 1 and ring2 configurations, respectively. In all configurations, the electrodes were placed near the spinal 
Table 1

Tissue conductivities

\begin{tabular}{llllll}
\hline \multirow{2}{*}{ Tissue } & \multirow{2}{*}{ Grey matter } & White matter & \multicolumn{2}{l}{$\begin{array}{l}\text { Cerebrospinal } \\
\text { fluid }\end{array}$} & \multirow{2}{*}{ KCl solution } \\
\cline { 3 - 4 } & & Longitudinal & Transverse & \\
\hline Conductivity $(\mathrm{S} / \mathrm{m})$ & 0.23 & 0.6 & 0.083 & 1.7 & 1.5214 \\
\hline
\end{tabular}

Table 2

Electrode parameters

\begin{tabular}{lll}
\hline Configuration & Surface area $\left(\mathrm{mm}^{2}\right)$ & Other parameters \\
\hline pin & 0.1256 & $1 \mathrm{~mm}$ long \\
Disc1 & 0.7854 & $0.2 \mathrm{~mm}$ thick \\
Disc2 & 3.14 & $0.2 \mathrm{~mm}$ thick \\
Disc3 & 0.7854 & $0.2 \mathrm{~mm}$ thick \\
Disc4 & 0.7854 & $0.2 \mathrm{~mm}$ thick \\
Ring1 & 2.512 & $0.5 \mathrm{~mm}$ wide \\
Ring2 & 5.024 & $1 \mathrm{~mm}$ wide \\
\hline
\end{tabular}

cord, but without direct contact with the tissue. The pin, disc and ring electrodes have been created already. The parameters of the electrodes are shown in Table 2.

Initially, the current density was set as $1 \mu \mathrm{A} / \mathrm{mm}^{2}$ for all configurations. According to the actual compensation effects, the necessary current densities were then calculated for different configurations. Finally, we conducted simulation in those conditions.

\subsection{Boundary condition}

In order to resolve the model, the following boundary conditions were imposed. The normal component of the current was set as 0 at the $\mathrm{XOZ}$ and YOZ planes. The electric potential of spinal cord section within $\mathrm{KCl}$ compartment was set as $-45 \mathrm{mV}$, and that at the injury site was set as $-10 \mathrm{mV}$. A certain current density was imposed to the anode electrodes. The surface of the cathode electrodes was set as ground and all other outer surfaces of the model were considered as insulated.

\subsection{Calculation of electric fields}

Through the Laplace equation $(\nabla(\sigma \nabla V)=0$, where $\sigma$ is the conductivity and $V$ is the electric potential), the electric potential was calculated. Then, the electric field was obtained through equation $E=-\nabla V$, where $E$ is the electric field. The calculations were performed by COMSOL Multiphysics.

\section{Results}

After SCI, the maximum longitudinal EF at the midpoint section of the spinal cord in the central compartment was $0.243 \mathrm{mV} / \mathrm{mm}$. When applying a current of $1 \mu \mathrm{A} / \mathrm{mm}^{2}$, through pin electrode, the maximum longitudinal $\mathrm{EF}$ was reduced to $0.203 \mathrm{mV} / \mathrm{mm}$. In order to compensate the injury potential completely, it is necessary to multiply the applied current density by 6.075 . 
Then, the magnitudes and distributions of EFs produced by the four disc configurations with applying $1 \mu \mathrm{A} / \mathrm{mm}^{2}$ were studied. With the disc1 configuration, the maximum longitudinal EF was reduced to $0.183 \mathrm{mV} / \mathrm{mm}$. The disc 2 configuration reduced the longitudinal $\mathrm{EF}$ to a greater extent; the maximum longitudinal EF was $0.07 \mathrm{mV} / \mathrm{mm}$. With the disc 3 and disc 4 configurations, the maximum longitudinal EFs were reduced to 0.16 and $0.12 \mathrm{mV} / \mathrm{mm}$, respectively. In order to compensate the injury potential completely, it would be necessary to apply current densities of $4,1.4,2.93$ and $1.98 \mu \mathrm{A} / \mathrm{mm}^{2}$ for disc1, disc2, disc 3 and disc 4 configurations, respectively.

When applying a current of $1 \mu \mathrm{A} / \mathrm{mm}^{2}$, with the ring 1 configuration, a maximum longitudinal EF of $-0.075 \mathrm{mV} / \mathrm{mm}$ was obtained. Meanwhile, the longitudinal EF decreased to $-0.2 \mathrm{mV} / \mathrm{mm}$ with the ring2 configuration at the same current density. In these cases, it would be necessary to reduce the applied current densities to 0.76 and $0.55 \mu \mathrm{A} / \mathrm{mm}^{2}$, respectively. These results are shown in Figure 2A.

Finally, the longitudinal EFs produced by different configurations with corresponding necessary current densities were compared. Figure 2B shows the longitudinal EFs obtained at the midpoint section of the spinal cord in the central compartment with those configurations. In order to investigate the compensation effects, intensities of the longitudinal EFs resulted from different configurations at the central, dorsal and ventral $(0.1 \mathrm{~mm}$ below the surface) regions (Figure 3$)$ were studied. These three regions correspond to the grey matter, dorsal and ventral axonal tracts, respectively. For the central region of the spinal cord, all configurations can compensate injury potential perfectly. At the ventral and dorsal regions, the compensation effects of different configurations vary. For the ventral region, the disc 3 , disc 4 , ring 1 and ring2 configurations have better compensation effects, whereas the disc 3 , disc 4 and ring 1 configurations led to overcompensation phenomenon. As for the dorsal region, only ring 1 and ring2 configurations have good compensation effects. Therefore, the best compensation approach is using ring electrode with an area of $5.024 \mathrm{~mm}^{2}$ and an applied current density of $0.55 \mu \mathrm{A} / \mathrm{mm}^{2}$.
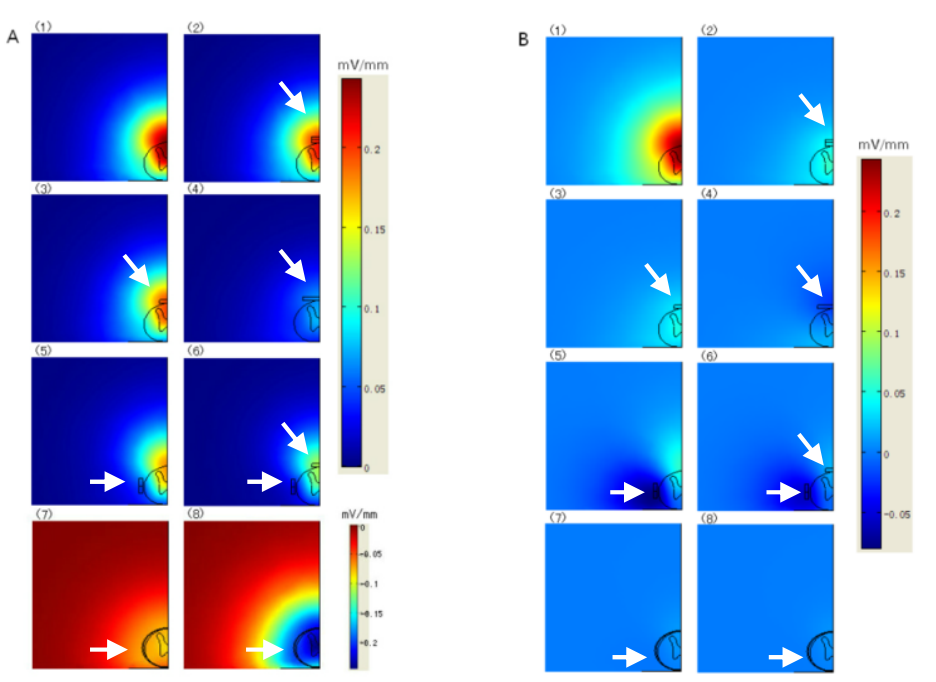

Fig. 2. Longitudinal EFs, produced by different configurations, at the midpoint section of the spinal cord in the central compartment. (1) Illustrations of EFs after SCI without stimulation. (2)-(8) Illustrations of EFs produced by pin, disc1, disc2, disc3, disc4, ring1 and ring2 configurations after SCI, respectively. In A, the current density was $1 \mu \mathrm{A} / \mathrm{mm}^{2}$ for all configurations, while in $\mathrm{B}$, the applied current densities for pin, disc1, disc2, disc3, disc4, ring1 and ring2 were 6.07, 4, 1.4, $2.93,1.98,0.76$ and $0.55 \mu \mathrm{A} / \mathrm{mm}^{2}$, respectively. The white arrows indicate the electrodes. 
(a)

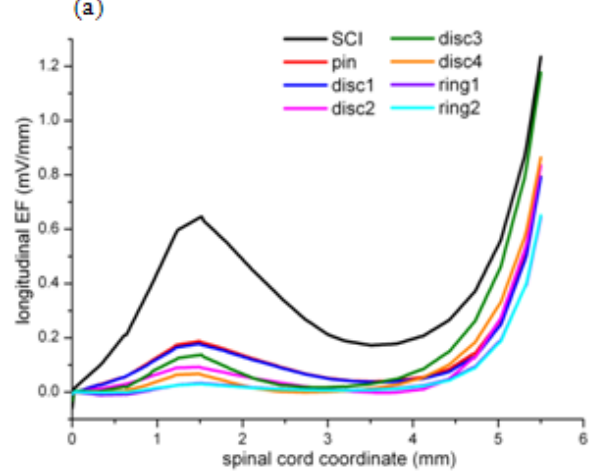

(c)

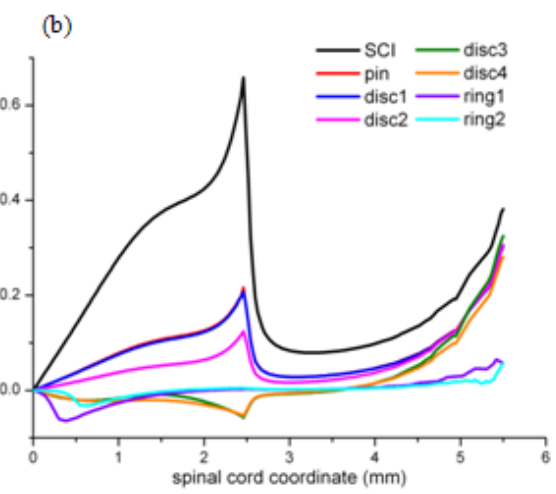

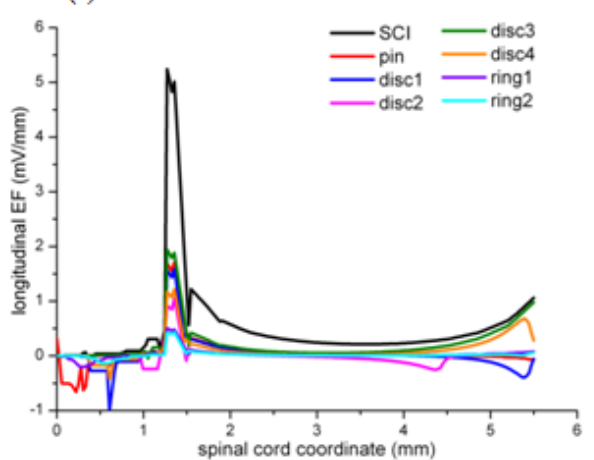

Fig. 3. Comparison of the longitudinal EFs produced at different levels of the mid-sagittal plane of the spinal cord with different configurations. (a)-(c) Illustrations of the longitudinal EFs at central, ventral and dorsal regions of the spinal cord, respectively. The applied current densities for pin, disc1, disc2, disc3, disc4, ring1 and ring2 were 6.07, 4, 1.4, 2.93, $1.98,0.76$ and 0.55 $\mu \mathrm{A} / \mathrm{mm}^{2}$, respectively.

\section{Discussion}

A 3D FEM of rat spinal cord was introduced to evaluate the compensation of injury potential by electrodes of different shapes, sizes and locations. The compensation effect was determined by the change of EFs, which was calculated with the model. The EFs generated by different electrodes have also been calculated by Gabriel [14], whose study focused on whether the EFs are adequate to promote axonal regrowth after SCI in human.

Direct current stimulation can produce detrimental changes in $\mathrm{pH}$, excessive heat and cytotoxic products with high electric currents, leading to tissue injury $[15,16]$, which is the main disadvantage of its use in SCI repairing. As the EF is directly proportional to the current density, an approach to diminish the current density is to enlarge the electrode area or to decrease the distance between anode and cathode. The simulation results showed that it is feasible to increase the electrode area and optimize its location in order to lower the current density. For instance, the $1 \mathrm{~mm}$ wide ring electrode compensated injury potential with a current density of $0.55 \mu \mathrm{A} / \mathrm{mm}^{2}$ applied. Larger electrodes will compensate injury potential with lower current densities, yet also bringing more difficulty for its experimental use.

According to the evaluation criterion of compensation effect, the magnitudes of longitudinal EFs should be almost reduced to 0 after appropriate electrical stimulation. In the simulation, the longitudinal EFs at the places close to sucrose gap were much greater than 0 . This may be caused by the idealization 
of the model, in which the compartments were considered completely separated. Due to the great difference between the conductivities of Krebs solution and sucrose, the electric potential had a significant change at the junction of the central compartment and the sucrose gap. Moreover, this produced a large $\mathrm{EF}$ at the junction.

Finally, it should be noticed that SCI is simply represented as a homogeneous electrical conductivity change, which cannot effectively reflect the real pathology of a traumatic lesion. Therefore, subtle differences of the EFs magnitudes between the actual measurement and the simulation results are expected. Moreover, the morphological changes of the injured spinal cord may induce further EF changes. Thus, in practical application, it is necessary to slightly adjust the current densities suggested by simulation results, according to the actual compensation effect.

\section{Conclusion}

Direct current stimulation may compensate injury potential and repair SCI, but it may cause tissue damage at high electric current. Thus, the determination of appropriate stimulation parameters is particularly important. Based on the results of finite element simulation, a principle was provided for this process, that is, ring electrode can compensate injury potential better with lower current density compared to pin and disc electrodes. Since the simulation was done with a simplified model, it is necessary to slightly adjust the current density in practical application.

\section{Acknowledgement}

This work was supported by the National Natural Science Foundation of China, No. 51177162.

\section{References}

[1] R.B. Borgens, L.F. Jaffe and M.J. Cohen, Large and persistent electrical currents enter the transected lamprey spinal cord, Proceedings of the National Academy of Sciences 77 (1980), 1209-1213.

[2] R.M. Goodman, K. Wachs, S. Keller et al., Spontaneous spinal cord injury potential in the rat, Neurosurgery 17 (1985), $757-759$.

[3] T. Khan, J. Myklebust, T. Swiontek et al., Electrical field distribution within the injured cat spinal cord injury potentials and field distribution, J. Neurotrauma. 11 (1994), 699-710.

[4] L. Leybaert and G. Deley, Interstitial and tissue cations and electrical potential after experimental spinal-cord injury, Experimental Brain Research 100 (1994), 369-375.

[5] M. Zuberi, P. Liu-Snyder, A. Ul Haque et al., Large naturally-produced electric currents and voltage traverse damaged mammalian spinal cord, J. Biol. Eng. 2 (2008), 17-27.

[6] S.Y. Pan, G.H. Zhang, X.L. Huo et al., Injury potentials associated with severity of acute spinal cord injury in an experimental rat model, Neural Regeneration Research 6 (2011), 1780-1785.

[7] G.H. Zhang, A.H. Wang, C. Zhang et al., Compensation for injury potential by electrical stimulation after acute spinal cord injury in rat, 35th Annual International Conference of the IEEE Engineering in Medicine and Biology Society, 2013, 3634-3637.

[8] Y. Shi, S. Kim, T.B. Huff et al., Effective repair of traumatically injured spinal cord by nanoscale block copolymer micelles, Nat. Nanotechnol. 5 (2009), 80-87.

[9] A. Nehrt, K. Hamann, H. Ouyang et al., Polyethylene glycol enhances axolemmal resealing following transection in cultured cells and in ex vivo spinal cord, J. Neurotrauma. 27 (2010), 151-161.

[10] Y. Cho, R. Shi, R. Borgens et al., Repairing the damaged spinal cord and brain with nanomedicine, Small 4 (2008), 1676-1681. 
[11] J.T. Maikos, Z. Qian, D. Metaxas et al., Finite element analysis of spinal cord injury in the rat, J. Neurotrauma. 25 (2008), $795-816$.

[12] J.J. Struijk, J. Holsheimer, V.B. Van et al., Epidural spinal cord stimulation: Calculation of field potentials with special reference to dorsal column nerve fibers, IEEE Transactions on Biomedical Engineering 38 (1991), 104-110.

[13] W. Sin and B. Coburn, Electrical stimulation of the spinal cord: A further analysis relating to anatomical factors and tissue properties, Medical and Biological Engineering and Computing 21 (1983), 264-269.

[14] G.R. Hernandez-Labrado, J.L. Polo, E. Lopez-Dolado et al., Spinal cord direct current stimulation: Finite element analysis of the electric field and current density, Med. Biol. Eng. Comput. 49 (2011), 417-429.

[15] A. Jackson, H. Yao, M. Brown et al., Anisotropic ion diffusivity in intervertebral disc: An electrical conductivity approach, Spine 31 (2006), 2783-2789.

[16] T.J. Swiontek, D. Maiman, A. Sances et al., Effect of electrical current on temperature and $\mathrm{pH}$ in cerebellum and spinal cord, Surg. Neurol. 14 (1980), 365-369. 